\title{
Heisenberg 型群上的强奇异卷积算子
}

献给陆善镇教授 75 华诞

\section{李洪全}

复旦大学数学科学学院, 上海 200433

E-mail: hongquan_li@fudan.edu.cn

收稿日期: 2013-05-30; 接受日期: 2013-08-28

国家自然科学基金 (批准号: 11171070) 资助项目

摘要 本文将给出 Heisenberg 型群上的一些强奇异卷积算子的 $L^{2}$ 有界性. 特别地, 本文的结果改进 并推广了 Laghi 和 Lyall 在 Heisenberg 群上的相应工作. 此外, 一些更简单、有效的技巧也在本文中 引入.

关键词 强奇异卷积算子 Heisenberg 型群 Korányi 度量

MSC (2010) 主题分类 $42 \mathrm{~B} 20,43 \mathrm{~A} 80$

\section{1 引言}

假设 $0<a<1, b \geqslant 1$, 考虑 $\mathbb{R}^{n}$ 上的乘子

$$
\widehat{T_{a, b} f}(\xi)=m_{a, b}(\xi) \hat{f}(\xi), \quad \xi \in \mathbb{R}^{n}, \quad f \in C_{0}^{\infty}\left(\mathbb{R}^{n}\right),
$$

其中

$$
m_{a, b}(\xi)=\psi(\xi) \mathrm{e}^{\mathrm{i}|\xi|^{a}}|\xi|^{-b}, \quad \psi \in C^{\infty}\left(\mathbb{R}^{n}\right), \quad \psi(\xi)= \begin{cases}0, & |\xi| \leqslant 1, \\ 1, & |\xi| \geqslant 2,\end{cases}
$$

则当 $1<p<+\infty$ 满足条件 $\left|\frac{1}{p}-\frac{1}{2}\right| \leqslant \frac{b}{n a}$ 时, $T_{a, b}$ 可以延拓成 $L^{p}\left(\mathbb{R}^{n}\right)$ 上的有界 (线性) 算子. 关于这 些结果及其来源, 参见文献 [1, 第 160 页] 和 [2-4].

注意到 $m_{a, b}(\xi)$ 不满足通常的 Mikhlin-Hörmander 乘子条件. 上述结论引发出一个非常自然的问 题: 在 Riemann 流形或者 Lie 群等合适的背景下, 考虑振动谱乘子

$$
m_{a, b}(\sqrt{-\Delta})=\varphi(\sqrt{-\Delta}) \mathrm{e}^{\mathrm{i}(-\Delta)^{a / 2}(-\Delta)^{-b / 2},}
$$

其中

$$
\varphi \in C^{\infty}\left(\mathbb{R}^{+}\right), \quad \varphi(r)= \begin{cases}0, & r \leqslant 1 \\ 1, & r \geqslant 2\end{cases}
$$


此处, $\Delta$ 为所考虑流形上的 Laplace 或者次 Laplace 算子. 例如, Giulini 和 Meda ${ }^{[5]}$ 在秩一非紧对 称空间上考虑了振动谱乘子; Alexopoulos ${ }^{[6]}$ 考虑了多项式增长 Lie 群以及具有非负 Ricci 曲率完备 Riemann 流形背景下的振动谱乘子; $\mathrm{Li}^{[7]}$ 考虑了紧基底雉流形上的振动谱乘子. 注意到文献 [5-7] 中 所研究的 $m_{a, b}(\sqrt{-\Delta})$ 并不局限于 $0<a<1$.

又由文献 [4], 卷积算子 $T_{a, b} f=f * K$ 的卷积核可表示为

$$
K(x)=C_{1}|x|^{-n-\alpha} \mathrm{e}^{\mathrm{i} C_{2}|x|^{-\beta}} \chi(|x|)+K_{1}(x),
$$

其中 $K_{1} \in L^{1}\left(\mathbb{R}^{n}\right), C_{1}, C_{2} \neq 0$ 为常数, $\chi \in C_{0}^{\infty}\left(\mathbb{R}^{n}\right)$ 在原点附近取值为 1 , 且

$$
\alpha=\frac{\frac{n}{2} a-b}{1-a}, \quad \beta=\frac{a}{1-a} .
$$

注意到

$$
S f=\left(|x|^{-n-\alpha} \mathrm{e}^{\mathrm{i}|x|^{-\beta}} \chi(|x|)\right) * f, \quad f \in C_{0}^{\infty}\left(\mathbb{R}^{n}\right)
$$

不属于经典的 Calderón-Zygmund 型奇异积分算子, 我们称为强奇异卷积算子. 由文献 [1, 第 160 页] 的结论知, 算子 $S$ 在 $L^{p}\left(\mathbb{R}^{n}\right)$ 上有界, 其中 $1<p<+\infty$ 满足条件

$$
\left|\frac{1}{p}-\frac{1}{2}\right| \leqslant \frac{1}{n} \frac{b}{a}=\frac{1}{2}-\frac{\alpha}{n \beta} .
$$

对应地, 我们也可以在一些流形上研究类似的强奇异积分算子. 事实上, 在文献 $[8,9]$ 中, 一些强奇异 卷积算子已经在 Heisenberg 群上研究过. 本文的目的是将文献 [8] 中的主要结果通过更简单、有效、 易懂的技巧推广至一般 Heisenberg 型群的情形. 为此, 我们首先回忆一些概念和记号.

简单地说 ${ }^{[10]}$, 一个 Heisenberg 型群 $H_{(2 n, m)}\left(m, n \in \mathbb{N}^{*}\right)$ 可看成 $\mathbb{R}^{2 n} \times \mathbb{R}^{m}$ 赋予群法则

$$
(x, t) \cdot\left(x^{\prime}, t^{\prime}\right)=\left(x+x^{\prime}, t+t^{\prime}+2^{-1}\left\langle x, U x^{\prime}\right\rangle\right)
$$

其中

$$
\begin{aligned}
& x^{\prime}, x=\left(x_{1}, \ldots, x_{2 n}\right) \in \mathbb{R}^{2 n}, \quad t^{\prime}, t=\left(t_{1}, \ldots, t_{m}\right) \in \mathbb{R}^{m}, \\
& \left\langle x, U x^{\prime}\right\rangle=\left(\left\langle x, U^{(1)} x^{\prime}\right\rangle, \ldots,\left\langle x, U^{(m)} x^{\prime}\right\rangle\right) \in \mathbb{R}^{m},
\end{aligned}
$$

此处, $U^{(i)}$ 为 $2 n \times 2 n$ 反对称正交矩阵且满足条件

$$
U^{(i)} U^{(j)}+U^{(j)} U^{(i)}=0, \quad \forall 1 \leqslant i<j \leqslant m .
$$

记 $U^{(j)}=\left(U_{k, l}^{(j)}\right)_{1 \leqslant k, l \leqslant 2 n}, 1 \leqslant j \leqslant m$, 在原点取值为 $\frac{\partial}{\partial x_{l}}(1 \leqslant l \leqslant 2 n)$ 的左右不变向量场分别为

$$
X_{l}=\frac{\partial}{\partial x_{l}}+\frac{1}{2} \sum_{j=1}^{m}\left(\sum_{k=1}^{2 n} x_{k} U_{k, l}^{(j)}\right) \frac{\partial}{\partial t_{j}}, \quad Y_{l}=\frac{\partial}{\partial x_{l}}+\frac{1}{2} \sum_{j=1}^{m}\left(\sum_{k=1}^{2 n} x_{k} U_{l, k}^{(j)}\right) \frac{\partial}{\partial t_{j}} .
$$

在原点取值为 $\frac{\partial}{\partial t_{j}}(1 \leqslant j \leqslant m)$ 的左右不变向量场均为

$$
T_{j}=\frac{\partial}{\partial t_{j}}
$$

498 
$H_{(2 n, m)}$ 上的次 Laplace 算子为 $\Delta=\sum_{l=1}^{2 n} X_{l}^{2}$.

$H_{(2 n, m)}$ 的原始 (等价) 定义见文献 [11]. 由文献 [11], $m$ 的选取是任意的; 但是, 当且仅当 $(2 n, m)$ 满足如下条件时可定义一个 Heisenberg 型群 $H_{(2 n, m)}$ :

$$
m<\rho(2 n)=8 j+2^{q}, \quad \text { 若 } 2 n=(2 l+1) 2^{4 j+q},
$$

此处, $l, j \in \mathbb{N}, 0 \leqslant q<3$. 当 $m=1$ 时, $H_{(2 n, 1)}$ 为我们所熟知的 Heisenberg 群.

自从 Kaplan ${ }^{[11]}$ 引入 $H_{(2 n, m)}$ 以来, 其上各种几何、分析问题的研究吸引了越来越多人的关注, 如文献 $[10,12-23]$ 及其引用的文献.

众所周知, $H_{(2 n, m)}$ 上的 Haar 测度为 $\mathbb{R}^{2 n} \times \mathbb{R}^{m}$ 上的 Lebesgue 测度, 其上最常用的两个度量为 Carnot-Carathéodory 度量 $d_{C C}$ (由 $\left\{X_{l} ; 1 \leqslant l \leqslant 2 n\right\}$ 定义) 和齐次度量 $d_{K}$ (由 Korányi 范数定义, 紧 密联系着 Green 函数, 即 $-\Delta$ 的基本解). 此外, $d_{C C}$ 和 $d_{K}$ 均为左不变度量, 即有

$$
d_{C C}\left(g g_{1}, g g_{2}\right)=d_{C C}\left(g_{1}, g_{2}\right), \quad d_{K}\left(g g_{1}, g g_{2}\right)=d_{K}\left(g_{1}, g_{2}\right), \quad \forall g, g_{1}, g_{2} \in H_{(2 n, m)} .
$$

约定 $o$ 为 $H_{(2 n, m)}$ 的单位元, 记 $d_{C C}(g)=d_{C C}(g, o), d_{K}(g)=d_{K}(g, o)$, 则有 (参见文献 [11])

$$
d_{K}(x, t)=\left(|x|^{4}+16|t|^{2}\right)^{1 / 4},
$$

其中,

$$
|x|=\left(\sum_{i=1}^{2 n} x_{i}^{2}\right)^{1 / 2}, \quad|t|=\left(\sum_{j=1}^{m} t_{j}^{2}\right)^{1 / 2} .
$$

令 $\mu(\varphi)=\frac{2 \varphi-\sin 2 \varphi}{2 \sin ^{2} \varphi}:(-\pi, \pi) \rightarrow \mathbb{R}$, 则 $\mu$ 为严格单调递增函数, 并且是从 $(-\pi, \pi)$ 到 $\mathbb{R}$ 的一个 微分同胚 (参见文献 $\left[24\right.$, 第 639 页]). 记 $\mu^{-1}$ 为其反函数, 若 $x \neq 0, d_{C C}^{2}(x, t)=\left(\frac{\theta}{\sin \theta}\right)^{2}|x|^{2}$, 其中 $\theta=\mu^{-1}\left(\frac{4 t}{|x|^{2}}\right)$ 而 $d_{C C}^{2}(0, t)=\pi|t|$ (参见文献 [23, 第 90 和 91 页] 和 [17]).

我们知道 (Heisenberg 群时的证明参见文献 [25], 但对一般的 Heisenberg 型群显然保持成立),

$$
d_{K}(x, t) \leqslant d_{C C}(x, t) \leqslant \sqrt{\pi} d_{K}(x, t), \quad \forall(x, t) \in H_{(2 n, m)},
$$

可以证明函数 $d_{C C}^{2}(x, t)$ 当 $x=0$ 时不是可微的. 因此, 我们下面考虑 $H_{(2 n, m)}$ 上的强奇异卷积算子时 主要局限于 $d_{K}$ 的情形. 具体而言, 我们考虑算子

$$
S f=K_{\alpha, \beta} * f, \quad f \in C_{0}^{\infty},
$$

其中 $K_{\alpha, \beta}=d^{-2 n-2-\alpha}(g) \mathrm{e}^{\mathrm{i} d^{-\beta}(g)} \chi(d(g)), \alpha \geqslant 0, \beta>0$, 此处, $\chi \in C_{0}^{\infty}(\mathbb{R})$ 在原点附近取值为 1 , 函数 $d(x, t) \in C^{\infty}\left(H_{(2 n, m)} \backslash\{o\}\right)$ 且存在常数 $L \geqslant 1$ 使得

$$
L^{-1} d_{K}(g) \leqslant d(g) \leqslant L d_{K}(g), \quad \forall g \in H_{(2 n, m)} .
$$

我们主要考虑算子 $S$ 的 $L^{2}$ 有界性, 注意到在 $K_{\alpha, \beta}$ 的定义中我们可以假定 $\chi$ 的紧支撑充分小 (否则仅需考虑作一个新的光滑截断即可). 我们的结果如下:

定理 1.1 若 $d(x, t)=d_{K}(x, \gamma t)$, 其中常数 $\gamma$ 满足 $0<\gamma^{2}<\frac{\beta+2}{2}\left[(2 \beta+5)+\sqrt{(2 \beta+5)^{2}-9}\right]$, 则 当 $\alpha \leqslant \frac{2 n+m}{2} \beta$ 时, $S$ 可延拓为 $L^{2}\left(H_{(2 n, m)}\right)$ 上的有界线性算子.

定理 1.2 若 $d^{2}(x, t)=\frac{1}{2}\left(|x|^{2}+\sqrt{|x|^{4}+4 \gamma^{2}|t|^{2}}\right.$ ), 则当常数 $0<\gamma^{2}<D(\beta)$ (定义见 (4.12), 特别 地, 有 $D(\beta) \geqslant 16+20 \sqrt{3}), \alpha \leqslant \frac{2 n+m}{2} \beta$ 时, $S$ 可延拓为 $L^{2}\left(H_{(2 n, m)}\right)$ 上的有界线性算子. 
注 1.1 (1) 在 Heisenberg 群的情形, 由文献 [9] 知, “ $\alpha \leqslant \frac{2 n+m}{2} \beta$ ” 也是定理 1.1 成立的必要 条件.

(2) 即使是在 Heisenberg 群的情形, 我们发现定理 1.2 明显优于文献 [8, 定理 2] 中的结果.

上述两个定理的证明思想是标准的 (参见文献 [26] 和 [8, 第 827-831 页]): 通过利用二进分 解、Cotlar 引理和尺度变换, 问题的关键和难点在于验证非退化条件

$$
\operatorname{det}\left(\mathbb{X}^{l}\left(\mathbb{X}^{r} d^{-\beta}(x, t)\right)\right) \neq 0, \quad \forall(x, t) \neq o,
$$

其中 $\mathbb{X}^{l}\left(\mathbb{X}^{r} \phi(x, t)\right)=\left(X_{i}^{l} X_{j}^{r} \phi(x, t)\right)_{1 \leqslant i, j \leqslant 2 n+m}$, 此处,

$$
\begin{aligned}
& \left(X_{1}^{l}, \ldots, X_{2 n}^{l}, X_{2 n+1}^{l}, \ldots, X_{2 n+m}^{l}\right)=\left(X_{1}, \ldots, X_{2 n}, T_{1}, \ldots, T_{m}\right), \\
& \left(X_{1}^{r}, \ldots, X_{2 n}^{r}, X_{2 n+1}^{r}, \ldots, X_{2 n+m}^{r}\right)=\left(Y_{1}, \ldots, Y_{2 n}, T_{1}, \ldots, T_{m}\right) .
\end{aligned}
$$

\section{$2 \operatorname{det}\left(\mathbb{X}^{l}\left(\mathbb{X}^{r} \phi(x, t)\right)\right)$ 的计算}

下面, 我们将大量使用分块矩阵运算, 我们也将 $x$ 看成列向量, $A^{\mathrm{T}}$ 表示矩阵 $A$ 的转置, 并记

$$
\begin{aligned}
& \nabla_{x}=\left(\frac{\partial}{\partial x_{1}}, \ldots, \frac{\partial}{\partial x_{2 n}}\right), \quad \nabla_{t}=\left(\frac{\partial}{\partial t_{1}}, \ldots, \frac{\partial}{\partial t_{m}}\right), \\
& U=\left(U^{(1)}, \ldots, U^{(m)}\right), \quad U x=\left(U^{(1)} x, \ldots, U^{(m)} x\right), \quad x^{\mathrm{T}} U^{\mathrm{T}}=(U x)^{\mathrm{T}}, \\
& \mathbb{X}^{l}=\left(X_{1}, \ldots, X_{2 n}, T_{1}, \ldots, T_{m}\right)^{\mathrm{T}}=\left(\begin{array}{cc}
I_{2 n} & \frac{1}{2} U x \\
O & I_{m}
\end{array}\right)\left(\nabla_{x}, \nabla_{t}\right)^{\mathrm{T}}, \\
& \mathbb{X}^{r}=\left(Y_{1}, \ldots, Y_{2 n}, T_{1}, \ldots, T_{m}\right)^{\mathrm{T}}=\left(\begin{array}{cc}
I_{2 n} & -\frac{1}{2} U x \\
O & I_{m}
\end{array}\right)\left(\nabla_{x}, \nabla_{t}\right)^{\mathrm{T}} .
\end{aligned}
$$

注意到

$$
\begin{aligned}
& \mathbb{X}^{l}\left(\mathbb{X}^{r} \phi(x, t)\right)=\mathbb{X}^{l} \circ\left(\mathbb{X}^{r} \phi(x, t)\right)^{\mathrm{T}} \\
& =\left(\begin{array}{cc}
I_{2 n} & \frac{1}{2} U x \\
O & I_{m}
\end{array}\right)\left(\nabla_{x}, \nabla_{t}\right)^{t} \circ\left(\nabla_{x} \phi(x, t), \nabla_{t} \phi(x, t)\right)\left(\begin{array}{cc}
I_{2 n} & O \\
-\frac{1}{2} x^{\mathrm{T}} U^{\mathrm{T}} & I_{m}
\end{array}\right) \\
& =\left(\begin{array}{cc}
I_{2 n} & \frac{1}{2} U x \\
O & I_{m}
\end{array}\right)\left(\nabla_{x}, \nabla_{t}\right)^{\mathrm{T}} \circ\left(\nabla_{x} \phi(x, t)-\frac{1}{2}\left(\nabla_{t} \phi(x, t)\right)\left(x^{\mathrm{T}} U^{\mathrm{T}}\right), \nabla_{t} \phi(x, t)\right) \\
& =\left(\begin{array}{cc}
I_{2 n} & \frac{1}{2} U x \\
O & I_{m}
\end{array}\right)\left(\begin{array}{cc}
\nabla_{x}^{\mathrm{T}}\left[\nabla_{x} \phi(x, t)-\frac{1}{2}\left(\nabla_{t} \phi(x, t)\right)\left(x^{\mathrm{T}} U^{\mathrm{T}}\right)\right] & \nabla_{x}^{\mathrm{T}} \nabla_{t} \phi(x, t) \\
\nabla_{t}^{\mathrm{T}}\left[\nabla_{x} \phi(x, t)-\frac{1}{2}\left(\nabla_{t} \phi(x, t)\right)\left(x^{\mathrm{T}} U^{\mathrm{T}}\right)\right] & \nabla_{t}^{\mathrm{T}} \nabla_{t} \phi(x, t)
\end{array}\right) .
\end{aligned}
$$

因此, 我们有

$$
\operatorname{det}\left(\mathbb{X}^{l}\left(\mathbb{X}^{r} \phi(x, t)\right)\right)=\operatorname{det}\left(\begin{array}{cc}
\nabla_{x}^{\mathrm{T}}\left[\nabla_{x} \phi(x, t)-\frac{1}{2}\left(\nabla_{t} \phi(x, t)\right)\left(x^{\mathrm{T}} U^{\mathrm{T}}\right)\right] & \nabla_{x}^{\mathrm{T}} \nabla_{t} \phi(x, t) \\
\nabla_{t}^{\mathrm{T}}\left[\nabla_{x} \phi(x, t)-\frac{1}{2}\left(\nabla_{t} \phi(x, t)\right)\left(x^{\mathrm{T}} U^{\mathrm{T}}\right)\right] & \nabla_{t}^{\mathrm{T}} \nabla_{t} \phi(x, t)
\end{array}\right) .
$$


既然,

$$
\nabla_{x}^{\mathrm{T}}\left[\nabla_{x} \phi(x, t)-\frac{1}{2}\left(\nabla_{t} \phi(x, t)\right)\left(x^{\mathrm{T}} U^{\mathrm{T}}\right)\right]=\nabla_{x}^{\mathrm{T}}\left(\nabla_{x} \phi(x, t)\right)-\frac{1}{2}\left[\nabla_{x}^{\mathrm{T}}\left(\nabla_{t} \phi(x, t)\right)\right] x^{\mathrm{T}} U^{\mathrm{T}}-\frac{1}{2}\left[\nabla_{t} \phi(x, t)\right] U^{\mathrm{T}},
$$

为了简化记号, 此处我们已经约定 $\left(t_{1}, \ldots, t_{m}\right) U^{\mathrm{T}}=\sum_{j=1}^{m} t_{j}\left(U^{(j)}\right)^{\mathrm{T}}=-\sum_{j=1}^{m} t_{j} U^{(j)}$,

$$
\nabla_{t}^{\mathrm{T}}\left[\nabla_{x} \phi(x, t)-\frac{1}{2}\left(\nabla_{t} \phi(x, t)\right)\left(x^{\mathrm{T}} U^{\mathrm{T}}\right)\right]=\nabla_{t}^{\mathrm{T}}\left(\nabla_{x} \phi(x, t)\right)-\frac{1}{2}\left[\nabla_{t}^{\mathrm{T}}\left(\nabla_{t} \phi(x, t)\right)\right] x^{\mathrm{T}} U^{\mathrm{T}},
$$

因此,

$$
\begin{aligned}
& \left(\begin{array}{ll}
\nabla_{x}^{\mathrm{T}}\left[\nabla_{x} \phi(x, t)-\frac{1}{2}\left(\nabla_{t} \phi(x, t)\right)\left(x^{\mathrm{T}} U^{\mathrm{T}}\right)\right] & \nabla_{x}^{\mathrm{T}} \nabla_{t} \phi(x, t) \\
\nabla_{t}^{\mathrm{T}}\left[\nabla_{x} \phi(x, t)-\frac{1}{2}\left(\nabla_{t} \phi(x, t)\right)\left(x^{\mathrm{T}} U^{\mathrm{T}}\right)\right] & \nabla_{t}^{\mathrm{T}} \nabla_{t} \phi(x, t)
\end{array}\right) \\
& \quad=\left(\begin{array}{ll}
\nabla_{x}^{\mathrm{T}}\left(\nabla_{x} \phi(x, t)\right)-\frac{1}{2}\left[\nabla_{t} \phi(x, t)\right] U^{\mathrm{T}} & \nabla_{x}^{\mathrm{T}} \nabla_{t} \phi(x, t) \\
\nabla_{t}^{\mathrm{T}}\left(\nabla_{x} \phi(x, t)\right) & \nabla_{t}^{\mathrm{T}} \nabla_{t} \phi(x, t)
\end{array}\right)\left(\begin{array}{cc}
I_{2 n} & O \\
-\frac{1}{2} x^{\mathrm{T}} U^{\mathrm{T}} & I_{m}
\end{array}\right) .
\end{aligned}
$$

于是, 我们得到

$$
\operatorname{det}\left(\mathbb{X}^{l}\left(\mathbb{X}^{r} \phi(x, t)\right)\right)=\operatorname{det}\left(\begin{array}{ll}
\nabla_{x}^{\mathrm{T}} \nabla_{x} \phi(x, t)-\frac{1}{2}\left[\nabla_{t} \phi(x, t)\right] U^{\mathrm{T}} & \nabla_{x}^{\mathrm{T}} \nabla_{t} \phi(x, t) \\
\nabla_{t}^{\mathrm{T}} \nabla_{x} \phi(x, t) & \nabla_{t}^{\mathrm{T}} \nabla_{t} \phi(x, t)
\end{array}\right) .
$$

下面, 我们总假设 $\phi=f \circ \rho$, 其中 $f$ 为 $\mathbb{R}^{+}$上的光滑实值函数, $\rho \in C^{\infty}\left(H_{(2 n, m)} \backslash\{o\}\right)$. 注意到

$$
\begin{aligned}
& \nabla_{x} \phi=f^{\prime} \nabla_{x} \rho, \quad \nabla_{t} \phi=f^{\prime} \nabla_{t} \rho, \quad \nabla_{x}^{\mathrm{T}} \nabla_{t} \phi=f^{\prime \prime}\left(\nabla_{x}^{\mathrm{T}} \rho\right)\left(\nabla_{t} \rho\right)+f^{\prime} \nabla_{x}^{\mathrm{T}}\left(\nabla_{t} \rho\right), \\
& \nabla_{t}^{\mathrm{T}} \nabla_{x} \phi=f^{\prime \prime}\left(\nabla_{t}^{\mathrm{T}} \rho\right)\left(\nabla_{x} \rho\right)+f^{\prime} \nabla_{t}^{\mathrm{T}}\left(\nabla_{x} \rho\right), \quad \nabla_{x}^{\mathrm{T}} \nabla_{x} \phi=f^{\prime \prime}\left(\nabla_{x}^{\mathrm{T}} \rho\right)\left(\nabla_{x} \rho\right)+f^{\prime} \nabla_{x}^{\mathrm{T}}\left(\nabla_{x} \rho\right), \\
& \nabla_{t}^{\mathrm{T}} \nabla_{t} \phi=f^{\prime \prime}\left(\nabla_{t}^{\mathrm{T}} \rho\right)\left(\nabla_{t} \rho\right)+f^{\prime} \nabla_{t}^{\mathrm{T}}\left(\nabla_{t} \rho\right) .
\end{aligned}
$$

此时, 由 (2.1) 有

$$
\operatorname{det}\left(\mathbb{X}^{l}\left(\mathbb{X}^{r} \phi(x, t)\right)\right)=\left|\begin{array}{cc}
f^{\prime \prime}\left(\nabla_{x}^{\mathrm{T}} \rho\right)\left(\nabla_{x} \rho\right)+f^{\prime} \nabla_{x}^{\mathrm{T}}\left(\nabla_{x} \rho\right)-\frac{1}{2} f^{\prime}\left(\nabla_{t} \rho\right) U^{\mathrm{T}} & f^{\prime \prime}\left(\nabla_{x}^{\mathrm{T}} \rho\right)\left(\nabla_{t} \rho\right)+f^{\prime} \nabla_{x}^{\mathrm{T}}\left(\nabla_{t} \rho\right) \\
f^{\prime \prime}\left(\nabla_{t}^{\mathrm{T}} \rho\right)\left(\nabla_{x} \rho\right)+f^{\prime} \nabla_{t}^{\mathrm{T}}\left(\nabla_{x} \rho\right) & f^{\prime \prime}\left(\nabla_{t}^{\mathrm{T}} \rho\right)\left(\nabla_{t} \rho\right)+f^{\prime} \nabla_{t}^{\mathrm{T}}\left(\nabla_{t} \rho\right)
\end{array}\right| .
$$

记上述矩阵为 $R_{1}$, 并记

$$
R_{1 *}=\left(\begin{array}{ll}
\nabla_{x}^{\mathrm{T}}\left(\nabla_{x} \rho\right)+\frac{1}{2} \sum_{i=1}^{m} \frac{\partial \rho}{\partial t_{i}} U^{(i)} & \nabla_{x}^{\mathrm{T}}\left(\nabla_{t} \rho\right) \\
\nabla_{t}^{\mathrm{T}}\left(\nabla_{x} \rho\right) & \nabla_{t}^{\mathrm{T}}\left(\nabla_{t} \rho\right)
\end{array}\right)
$$

我们有

$$
R_{1}=f^{\prime}\left[R_{1 *}+\frac{f^{\prime \prime}}{f^{\prime}}\left(\nabla_{x} \rho, \nabla_{t} \rho\right)^{\mathrm{T}}\left(\nabla_{x} \rho, \nabla_{t} \rho\right)\right]
$$

综上得

$$
\operatorname{det}\left(\mathbb{X}^{l}\left(\mathbb{X}^{r} \phi(x, t)\right)\right)=\left(f^{\prime}\right)^{2 n+m} \operatorname{det}\left(R_{1 *}+\frac{f^{\prime \prime}}{f^{\prime}}\left(\nabla_{x} \rho, \nabla_{t} \rho\right)^{\mathrm{T}}\left(\nabla_{x} \rho, \nabla_{t} \rho\right)\right) .
$$

在结束本节之前, 我们给出一个本文中将反复运用的初等引理: 
引理 2.1 若 $A$ 和 $D$ 分别为 $k \times k$ 和 $l \times l$ 方阵, $B$ 和 $C$ 分别为 $k \times l$ 和 $l \times k$ 矩阵, 则

$$
\begin{aligned}
\left|\begin{array}{ll}
A & B \\
C & D
\end{array}\right| & =|A|\left|D-C A^{-1} B\right| \quad \text { (若 } A \text { 可逆) } \\
& =|D|\left|A-B D^{-1} C\right| \text { (若 } D \text { 可逆). }
\end{aligned}
$$

\section{3 定理 1.1 的证明}

在定理 1.1 的假设条件下有 $\rho(x, t)=\rho_{1}(x)+\rho_{2}(t)$, 此时, 我们有

$$
R_{1 *}=\left(\begin{array}{cc}
R_{2} & O \\
O & \nabla_{t}^{\mathrm{T}}\left(\nabla_{t} \rho_{2}\right)
\end{array}\right),
$$

其中 $R_{2}=\nabla_{x}^{\mathrm{T}}\left(\nabla_{x} \rho_{1}\right)+\frac{1}{2} \sum_{i=1}^{m} \frac{\partial \rho_{2}}{\partial t_{i}} U^{(i)}$. 当 $R_{1 *}$ 可逆时, 等价地, 若 $R_{2}$ 和 $\nabla_{t}^{\mathrm{T}}\left(\nabla_{t} \rho_{2}\right)$ 同时可逆 (下面 我们将看到这仅分别需 $(x, t) \neq o$ 和 $\left.\gamma^{2}>0\right)$, 有

$$
R_{1 *}^{-1}=\left(\begin{array}{cc}
R_{2}^{-1} & O \\
O & \left(\nabla_{t}^{\mathrm{T}}\left(\nabla_{t} \rho_{2}\right)\right)^{-1}
\end{array}\right)
$$

由 (2.3) 和 $(2.4)$ 导出

$$
\begin{aligned}
\operatorname{det}\left(\mathbb{X}^{l}\left(\mathbb{X}^{r} \phi(x, t)\right)\right)= & \left(f^{\prime}\right)^{2 n+m} \operatorname{det}\left(R_{1 *}\right)\left[1+\frac{f^{\prime \prime}}{f^{\prime}}\left(\nabla_{x} \rho_{1}, \nabla_{t} \rho_{2}\right) R_{1 *}^{-1}\left(\nabla_{x} \rho_{1}, \nabla_{t} \rho_{2}\right)^{\mathrm{T}}\right] \\
= & \left(f^{\prime}\right)^{2 n+m} \operatorname{det}\left(\nabla_{t}^{\mathrm{T}}\left(\nabla_{t} \rho_{2}\right)\right) \operatorname{det}\left(R_{2}\right) \\
& \times\left\{1+\frac{f^{\prime \prime}}{f^{\prime}}\left[\left(\nabla_{x} \rho_{1}\right) R_{2}^{-1}\left(\nabla_{x} \rho_{1}\right)^{\mathrm{T}}+\left(\nabla_{t} \rho_{2}\right)\left(\nabla_{t}^{\mathrm{T}}\left(\nabla_{t} \rho_{2}\right)\right)^{-1}\left(\nabla_{t} \rho_{2}\right)^{\mathrm{T}}\right]\right\} .
\end{aligned}
$$

特别地, 当 $\rho_{1}(x)=|x|^{4}, \rho_{2}(t)=16 \gamma^{2}|t|^{2}$ 时, 有

$$
\begin{aligned}
& \nabla_{t} \rho_{2}=32 \gamma^{2} t^{\mathrm{T}}, \quad \nabla_{x} \rho_{1}=4|x|^{2} x^{\mathrm{T}}, \quad \nabla_{t}^{\mathrm{T}}\left(\nabla_{t} \rho_{2}\right)=32 \gamma^{2} I_{m}, \quad \nabla_{x}^{\mathrm{T}}\left(\nabla_{x} \rho_{1}\right)=4|x|^{2} I_{2 n}+8 x x^{\mathrm{T}}, \\
& R_{2}=4|x|^{2} I_{2 n}+8 x x^{\mathrm{T}}+16 \gamma^{2} \sum_{i=1}^{m} t_{i} U^{(i)}=4\left[\left(|x|^{2} I_{2 n}+4 \gamma^{2} \sum_{i=1}^{m} t_{i} U^{(i)}\right)+2 x x^{\mathrm{T}}\right]=4 R_{2 *} .
\end{aligned}
$$

我们首先计算 $\left(\nabla_{x} \rho_{1}\right) R_{2}^{-1}\left(\nabla_{x} \rho_{1}\right)^{\mathrm{T}}$. 由 $(2.4)$ 有

$$
\left(\nabla_{x} \rho_{1}\right) R_{2}^{-1}\left(\nabla_{x} \rho_{1}\right)^{\mathrm{T}}=-\left|R_{2}\right|^{-1}\left|\begin{array}{cc}
R_{2} & \left(\nabla_{x} \rho_{1}\right)^{\mathrm{T}} \\
\nabla_{x} \rho_{1} & 0
\end{array}\right| .
$$

既然我们有

$$
\begin{aligned}
\left|\begin{array}{cc}
R_{2} & \left(\nabla_{x} \rho_{1}\right)^{\mathrm{T}} \\
\nabla_{x} \rho_{1} & 0
\end{array}\right| & =\left|\begin{array}{cc}
4 R_{2 *} & 4|x|^{2} x \\
4|x|^{2} x^{\mathrm{T}} & 0
\end{array}\right|=4^{2 n+1}|x|^{4}\left|\begin{array}{cc}
R_{2 *} & x \\
x^{\mathrm{T}} & 0
\end{array}\right| \\
& =4^{2 n+1}|x|^{4}\left|\begin{array}{cc}
\left(|x|^{2} I_{2 n}+4 \gamma^{2} \sum_{i=1}^{m} t_{i} U^{(i)}\right)+2 x x^{\mathrm{T}} & x \\
x^{\mathrm{T}} & 0
\end{array}\right|,
\end{aligned}
$$


以及

$$
\left(\begin{array}{cc}
\left(|x|^{2} I_{2 n}+4 \gamma^{2} \sum_{i=1}^{m} t_{i} U^{(i)}\right)+2 x x^{\mathrm{T}} & x \\
x^{\mathrm{T}} & 0
\end{array}\right)\left(\begin{array}{cc}
I_{2 n} & O \\
-2 x^{\mathrm{T}} & 1
\end{array}\right)=\left(\begin{array}{cc}
|x|^{2} I_{2 n}+4 \gamma^{2} \sum_{i=1}^{m} t_{i} U^{(i)} & x \\
x^{\mathrm{T}} & 0
\end{array}\right),
$$

因此, 当 $(x, t) \neq o, \gamma^{2}>0$ 时,

$$
\left|\begin{array}{cc}
R_{2} & \left(\nabla_{x} \rho_{1}\right)^{\mathrm{T}} \\
\nabla_{x} \rho_{1} & 0
\end{array}\right|=4^{2 n+1}|x|^{4}\left|\begin{array}{cc}
|x|^{2} I_{2 n}+4 \gamma^{2} \sum_{i=1}^{m} t_{i} U^{(i)} & x \\
x^{\mathrm{T}} & 0
\end{array}\right|=4^{2 n+1}|x|^{4} \operatorname{det}\left(R_{3}\right)\left(-x^{\mathrm{T}} R_{3}^{-1} x\right),
$$

在最后一个等式中我们已经令

$$
R_{3}=|x|^{2} I_{2 n}+4 \gamma^{2} \sum_{i=1}^{m} t_{i} U^{(i)}
$$

并利用了 (2.4). 注意到由 (1.1) 有

$$
R_{3} R_{3}^{\mathrm{T}}=|x|^{4} I_{2 n}-16 \gamma^{4}\left(\sum_{i=1}^{m} t_{i} U^{(i)}\right)^{2}=|x|^{4} I_{2 n}+16 \gamma^{4}|t|^{2} I_{2 n}=\left(|x|^{4}+16 \gamma^{4}|t|^{2}\right) I_{2 n},
$$

由此有

$$
R_{3}^{-1}=\left(|x|^{4}+16 \gamma^{4}|t|^{2}\right)^{-1}\left(|x|^{2} I_{2 n}-4 \gamma^{2} \sum_{i=1}^{m} t_{i} U^{(i)}\right), \quad \operatorname{det}\left(R_{3}\right)=\left(|x|^{4}+16 \gamma^{4}|t|^{2}\right)^{n}
$$

从而,

$$
\begin{aligned}
\left|\begin{array}{cc}
R_{2} & \left(\nabla_{x} \rho_{1}\right)^{\mathrm{T}} \\
\nabla_{x} \rho_{1} & 0
\end{array}\right| & =4^{2 n+1}|x|^{4}\left(|x|^{4}+16 \gamma^{4}|t|^{2}\right)^{n-1}\left[-x^{\mathrm{T}}\left(|x|^{2} I_{2 n}-4 \gamma^{2} \sum_{i=1}^{m} t_{i} U^{(i)}\right) x\right] \\
& =-4^{2 n+1}|x|^{8}\left(|x|^{4}+16 \gamma^{4}|t|^{2}\right)^{n-1} \text { (由于 } U^{(i)}(1 \leqslant i \leqslant m \text { ) 为反对称矩阵). }
\end{aligned}
$$

因此, 我们得到

$$
\left(\nabla_{x} \rho_{1}\right) R_{2}^{-1}\left(\nabla_{x} \rho_{1}\right)^{\mathrm{T}}=4^{2 n+1}|x|^{8}\left(|x|^{4}+16 \gamma^{4}|t|^{2}\right)^{n-1}\left|R_{2}\right|^{-1} .
$$

回忆 $R_{2}=4 R_{2 *}=4\left(R_{3}+2 x x^{\mathrm{T}}\right)$, 由 $(2.4)$ 和 $U^{(i)}(1 \leqslant i \leqslant m)$ 的反对称性有

$$
\operatorname{det}\left(R_{2}\right)=4^{2 n} \operatorname{det}\left(R_{3}\right)\left(1+2 x^{\mathrm{T}} R_{3}^{-1} x\right)=4^{2 n}\left(|x|^{4}+16 \gamma^{4}|t|^{2}\right)^{n}\left(1+\frac{2|x|^{4}}{|x|^{4}+16 \gamma^{4}|t|^{2}}\right) .
$$

于是, 我们有

$$
\left(\nabla_{x} \rho_{1}\right) R_{2}^{-1}\left(\nabla_{x} \rho_{1}\right)^{\mathrm{T}}=4|x|^{8}\left(3|x|^{4}+16 \gamma^{4}|t|^{2}\right)^{-1} .
$$

综上, 我们有

$$
\begin{aligned}
\operatorname{det}\left(\mathbb{X}^{l}\left(\mathbb{X}^{r} \phi(x, t)\right)\right)= & \left(f^{\prime}\right)^{2 n+m}\left(32 \gamma^{2}\right)^{m} 4^{2 n}\left(|x|^{4}+16 \gamma^{4}|t|^{2}\right)^{n-1} \times\left(3|x|^{4}+16 \gamma^{4}|t|^{2}\right) \\
& \times\left\{1+\frac{f^{\prime \prime}}{f^{\prime}}\left[\frac{4|x|^{8}}{3|x|^{4}+16 \gamma^{4}|t|^{2}}+32 \gamma^{2}|t|^{2}\right]\right\}
\end{aligned}
$$


显然, 当 $\gamma^{2}>0$ 时, 为了保证 $\operatorname{det}\left(\mathbb{X}^{l}\left(\mathbb{X}^{r} \phi(x, t)\right)\right)$ 在原点以外非零, 当且仅当

$$
\Psi=\left(3|x|^{4}+16 \gamma^{4}|t|^{2}\right)+\frac{f^{\prime \prime}}{f^{\prime}}\left[4|x|^{8}+32 \gamma^{2}|t|^{2}\left(3|x|^{4}+16 \gamma^{4}|t|^{2}\right)\right]
$$

在原点以外非零.

在定理 1.1 的情形, 我们选取 $f(\lambda)=\lambda^{-\frac{\beta}{4}}$, 有

$$
f^{\prime}(\lambda)=-\frac{\beta}{4} \lambda^{-\frac{\beta}{4}-1}, \quad f^{\prime \prime}(\lambda)=\frac{\beta}{4}\left(1+\frac{\beta}{4}\right) \lambda^{-\frac{\beta}{4}-2}, \quad \frac{f^{\prime \prime}}{f^{\prime}}=-\left(1+\frac{\beta}{4}\right) \lambda^{-1},
$$

此处, $\lambda=\rho(x, t)=\rho_{1}(x)+\rho_{2}(t)=|x|^{4}+16 \gamma^{2}|t|^{2}$. 问题归结为

$$
\begin{aligned}
\Xi & =\left(3|x|^{4}+16 \gamma^{4}|t|^{2}\right)\left(|x|^{4}+16 \gamma^{2}|t|^{2}\right)-(\beta+4)\left[|x|^{8}+8 \gamma^{2}|t|^{2}\left(3|x|^{4}+16 \gamma^{4}|t|^{2}\right)\right] \\
& =-\left\{(\beta+1)|x|^{8}+\left[24 \gamma^{2}(\beta+2)-16 \gamma^{4}\right]|x|^{4}|t|^{2}+128 \gamma^{6}(\beta+2)|t|^{4}\right\}
\end{aligned}
$$

在原点以外非零. 首先, 当 $24 \gamma^{2}(\beta+2)-16 \gamma^{4} \geqslant 0$, 即 $\gamma^{2} \leqslant \frac{3}{2}(\beta+2)$ 时, $\Xi$ 仅在原点处为零. 当 $24 \gamma^{2}(\beta+2)-16 \gamma^{4}<0$ 时, 将 $\Xi$ 看成是 $|x|^{4}$ 或者 $|t|^{2}$ 的二次多项式, 需要二次判别式

$$
\begin{aligned}
\Delta & =\left[24 \gamma^{2}(\beta+2)-16 \gamma^{4}\right]^{2}-4(\beta+1) \times 128 \gamma^{6}(\beta+2) \\
& =64 \gamma^{4}\left\{4 \gamma^{4}-4(\beta+2)(2 \beta+5) \gamma^{2}+9(\beta+2)^{2}\right\}<0 .
\end{aligned}
$$

为此,

$$
\frac{\beta+2}{2}\left[(2 \beta+5)-\sqrt{(2 \beta+5)^{2}-9}\right]<\gamma^{2}<\frac{\beta+2}{2}\left[(2 \beta+5)+\sqrt{(2 \beta+5)^{2}-9}\right] .
$$

综上, 当

$$
\gamma^{2}<\frac{\beta+2}{2}\left[(2 \beta+5)+\sqrt{(2 \beta+5)^{2}-9}\right]
$$

时, $\operatorname{det}\left(\mathbb{X}^{l}\left(\mathbb{X}^{r} \phi(x, t)\right)\right)$ 在原点外非零, 定理 1.1 成立.

\section{4 定理 1.2 的证明}

我们记

$$
\rho=\frac{1}{2}\left(|x|^{2}+\sqrt{|x|^{4}+4 \gamma^{2}|t|^{2}}\right), \quad H=\frac{1}{\sqrt{|x|^{4}+4 \gamma^{2}|t|^{2}}} .
$$

首先, 我们有

$$
\begin{aligned}
& \nabla_{x} \rho=\left(1+|x|^{2} H\right) x^{\mathrm{T}}, \quad \nabla_{x}^{\mathrm{T}}\left(\nabla_{x} \rho\right)=\left(1+|x|^{2} H\right) I_{2 n}+2 H x x^{\mathrm{T}}-2|x|^{4} H^{3} x x^{\mathrm{T}}, \\
& \nabla_{t} \rho=2 \gamma^{2} H t^{\mathrm{T}}, \quad \nabla_{t}^{\mathrm{T}}\left(\nabla_{t} \rho\right)=2 \gamma^{2} H I_{m}-8 \gamma^{4} H^{3} t t^{\mathrm{T}}, \quad \nabla_{x}^{\mathrm{T}}\left(\nabla_{t} \rho\right)=-4 \gamma^{2}|x|^{2} H^{3} x t^{\mathrm{T}} .
\end{aligned}
$$

为了简化记号, 我们记

$$
\begin{aligned}
& W=\left(1+|x|^{2} H\right) I_{2 n}+\gamma^{2} H \sum_{i=1}^{m} t_{i} U^{(i)}, \quad R_{3}=W+2 H x x^{\mathrm{T}}, \\
& R_{2}=\left(\begin{array}{cc}
R_{3} & O \\
O & 2 \gamma^{2} H I_{m}
\end{array}\right), \quad V_{1}=\left(|x|^{2} x^{\mathrm{T}} \quad 2 \gamma^{2} t^{\mathrm{T}}\right) .
\end{aligned}
$$


将 (4.2) 代入 (2.2) 得

$$
R_{1 *}=R_{2}-2 H^{3}\left(\begin{array}{cc}
|x|^{4} x x^{\mathrm{T}} & 2 \gamma^{2}|x|^{2} x t^{\mathrm{T}} \\
2 \gamma^{2}|x|^{2} t x^{\mathrm{T}} & 4 \gamma^{4} t t^{\mathrm{T}}
\end{array}\right)=R_{2}-2 H^{3} V_{1}^{\mathrm{T}} V_{1} .
$$

由假设条件 (1.1) 有

$$
\begin{aligned}
W W^{\mathrm{T}} & =\left(\left(1+|x|^{2} H\right) I_{2 n}+\gamma^{2} H \sum_{i=1}^{m} t_{i} U^{(i)}\right)\left(\left(1+|x|^{2} H\right) I_{2 n}-\gamma^{2} H \sum_{i=1}^{m} t_{i} U^{(i)}\right) \\
& =\left[\left(1+|x|^{2} H\right)^{2}+\gamma^{4}|t|^{2} H^{2}\right] I_{2 n} .
\end{aligned}
$$

从而, 我们有 $\operatorname{det}(W)=\left[\left(1+|x|^{2} H\right)^{2}+\gamma^{4}|t|^{2} H^{2}\right]^{n}$,

$$
\begin{aligned}
& W^{-1}=\left[\left(1+|x|^{2} H\right)^{2}+\gamma^{4}|t|^{2} H^{2}\right]^{-1}\left(\left(1+|x|^{2} H\right) I_{2 n}-\gamma^{2} H \sum_{i=1}^{m} t_{i} U^{(i)}\right), \\
& 2 H x^{\mathrm{T}} W^{-1} x=\frac{2 H\left(1+|x|^{2} H\right)|x|^{2}}{\left(1+|x|^{2} H\right)^{2}+\gamma^{4}|t|^{2} H^{2}}=\frac{4|x|^{2} \rho}{4 \rho^{2}+\gamma^{4}|t|^{2}} \quad \text { (利用 } U^{(i)} \text { 的反对称性). }
\end{aligned}
$$

由 $R_{3}$ 的定义和 (2.4), 我们得到

$$
\operatorname{det}\left(R_{3}\right)=\operatorname{det}(W)\left(1+2 H x^{\mathrm{T}} W^{-1} x\right)=\left[\left(1+|x|^{2} H\right)^{2}+\gamma^{4}|t|^{2} H^{2}\right]^{n} \frac{4 \rho^{2}+\gamma^{4}|t|^{2}+4|x|^{2} \rho}{4 \rho^{2}+\gamma^{4}|t|^{2}} .
$$

显然, 当 $(x, t) \neq o$ 时, $R_{3}$ 可逆, 我们下面求 $R_{3}^{-1}$. 为此, 需要如下初等引理:

引理 4.1 若 $k$ 维列向量 $u$ 和 $v$ 满足 $1+v^{\mathrm{T}} u \neq 0$, 则

$$
\left(I_{k}+u v^{\mathrm{T}}\right)^{-1}=I_{k}-\frac{1}{1+v^{\mathrm{T}} u} u v^{\mathrm{T}} .
$$

由 (4.6) 有

$$
R_{3}^{-1}=\left(W+2 H x x^{\mathrm{T}}\right)^{-1}=\left(I_{2 n}+2 H W^{-1} x x^{\mathrm{T}}\right)^{-1} W^{-1}=W^{-1}-\frac{2 H W^{-1} x x^{\mathrm{T}} W^{-1}}{1+2 H x^{\mathrm{T}} W^{-1} x} .
$$

由 $R_{2}$ 的定义 (见 (4.3)), 有

$$
\operatorname{det}\left(R_{2}\right)=\left(2 \gamma^{2} H\right)^{m} \operatorname{det}\left(R_{3}\right)=\left(2 \gamma^{2} H\right)^{m}\left[\left(1+|x|^{2} H\right)^{2}+\gamma^{4}|t|^{2} H^{2}\right]^{n} \frac{4 \rho^{2}+\gamma^{4}|t|^{2}+4|x|^{2} \rho}{4 \rho^{2}+\gamma^{4}|t|^{2}} .
$$

特别地, 当 $(x, t) \neq o$ 时, $R_{2}$ 可逆, 且有

$$
R_{2}^{-1}=\left(\begin{array}{cc}
R_{3}^{-1} & O \\
O & \frac{1}{2 \gamma^{2} H} I_{m}
\end{array}\right)=\left(\begin{array}{cc}
W^{-1}-\frac{2 H}{1+2 H x^{\mathrm{T}} W^{-1} x} W^{-1} x x^{\mathrm{T}} W^{-1} & O \\
O & \frac{1}{2 \gamma^{2} H} I_{m}
\end{array}\right) .
$$

于是, (4.4) 和 (2.4) 导出

$$
\operatorname{det}\left(R_{1 *}\right)=\operatorname{det}\left(R_{2}\right)\left[1-2 H^{3} V_{1} R_{2}^{-1} V_{1}^{\mathrm{T}}\right]=\operatorname{det}\left(R_{2}\right)\left(1-2 H^{3}|x|^{4} x^{\mathrm{T}} R_{3}^{-1} x-4 \gamma^{2} H^{2}|t|^{2}\right) .
$$

注意到

$$
x^{\mathrm{T}} R_{3}^{-1} x=x^{\mathrm{T}} W^{-1} x-\frac{2 H\left(x^{\mathrm{T}} W^{-1} x\right)^{2}}{1+2 H x^{\mathrm{T}} W^{-1} x}=\frac{x^{\mathrm{T}} W^{-1} x}{1+2 H x^{\mathrm{T}} W^{-1} x}=H^{-1} \frac{2 \rho|x|^{2}}{4 \rho^{2}+\gamma^{4}|t|^{2}+4|x|^{2} \rho},
$$


最后一个等式来自于 (4.5). 由 (4.1) 有

$$
1-2 H^{3} V_{1} R_{2}^{-1} V_{1}^{\mathrm{T}}=1-4 \gamma^{2}|t|^{2} H^{2}-2 H^{3}|x|^{4} x^{\mathrm{T}} R_{3}^{-1} x=|x|^{4} H^{2} \frac{4 \rho^{2}+\gamma^{4}|t|^{2}}{4 \rho^{2}+\gamma^{4}|t|^{2}+4|x|^{2} \rho} .
$$

将上式代入 (4.9), 并利用 (4.7) 得

$$
\operatorname{det}\left(R_{1 *}\right)=\left[\left(1+|x|^{2} H\right)^{2}+\gamma^{4}|t|^{2} H^{2}\right]^{n}\left(2 \gamma^{2} H\right)^{m}|x|^{4} H^{2},
$$

特别地, $R_{1 *}$ 可逆当且仅当 $x \neq o$. 此时, 由 (4.4) 和 (4.6) 可得

$$
R_{1 *}^{-1}=R_{2}^{-1}+\frac{2 H^{3} R_{2}^{-1} V_{1}^{\mathrm{T}} V_{1} R_{2}^{-1}}{1-2 H^{3} V_{1} R_{2}^{-1} V_{1}^{\mathrm{T}}}=R_{2}^{-1}+\frac{2 H}{|x|^{4}} \frac{4 \rho^{2}+\gamma^{4}|t|^{2}+4|x|^{2} \rho}{4 \rho^{2}+\gamma^{4}|t|^{2}} R_{2}^{-1} V_{1}^{\mathrm{T}} V_{1} R_{2}^{-1} .
$$

在定理 1.2 的假设条件下, 我们取 $f(\lambda)=\lambda^{-\frac{\beta}{2}}$. 显然,

$$
f^{\prime}(\rho)=\left(-\frac{\beta}{2}\right) \rho^{-\frac{\beta}{2}-1}, \quad f^{\prime \prime}(\rho)=\frac{\beta}{2}\left(\frac{\beta}{2}+1\right) \rho^{-\frac{\beta}{2}-2}, \quad \frac{f^{\prime \prime}}{f^{\prime}}=-\frac{\beta+2}{2} \frac{1}{\rho} .
$$

下面分两种情形讨论:

情形 $1 x=o$ 且 $t \neq 0$. 此时, 为了证明 $\operatorname{det}\left(\mathbb{X}^{l} \mathbb{X}^{r} \phi(x, t)\right) \neq 0$, 由 (2.3)、(4.4)、(4.3) 和 $R_{3}$ 的可逆 性, 仅需证明

$$
\frac{|\gamma|}{|t|}\left(I_{m}-\frac{\beta+4}{2} \frac{1}{|t|^{2}} t t^{\mathrm{T}}\right)
$$

可逆. 由 (4.6), 我们剩下证明 $1 \neq \frac{\beta+4}{2}$, 而这是显然的, 因为 $\beta>0$.

情形 $2 x \neq o$. 此时, 令 $V_{2}=\left(\nabla_{x} \rho, \nabla_{t} \rho\right)=\left(\left(1+|x|^{2} H\right) x^{\mathrm{T}}, 2 \gamma^{2} H t^{\mathrm{T}}\right)$, 由 (2.3) 和 (2.4) 有

$$
\operatorname{det}\left(\mathbb{X}^{l} \mathbb{X}^{r} \phi(x, t)\right)=\left(f^{\prime}\right)^{2 n+m} \operatorname{det}\left(R_{1 *}+\frac{f^{\prime \prime}}{f^{\prime}} V_{2}^{\mathrm{T}} V_{2}\right)=\left(f^{\prime}\right)^{2 n+m} \operatorname{det}\left(R_{1 *}\right)\left[1+\frac{f^{\prime \prime}}{f^{\prime}} V_{2} R_{1 *}^{-1} V_{2}^{\mathrm{T}}\right],
$$

利用 (4.11), 我们剩下计算 $V_{2} R_{2}^{-1} V_{2}^{\mathrm{T}}$ 和 $V_{2} R_{2}^{-1} V_{1}^{\mathrm{T}}$. 由 (4.8)、(4.10) 和 (4.1) 推导出

$$
\begin{aligned}
& V_{2} R_{2}^{-1} V_{2}^{\mathrm{T}}=\left(1+|x|^{2} H\right)^{2} x^{\mathrm{T}} R_{3}^{-1} x+2 \gamma^{2} H|t|^{2}=2 H\left(\frac{4|x|^{2} \rho^{3}}{4 \rho^{2}+4|x|^{2} \rho+\gamma^{4}|t|^{2}}+\gamma^{2}|t|^{2}\right), \\
& V_{2} R_{2}^{-1} V_{1}^{\mathrm{T}}=\left(1+|x|^{2} H\right)|x|^{2} x^{\mathrm{T}} R_{3}^{-1} x+2 \gamma^{2}|t|^{2}=\frac{4|x|^{4} \rho^{2}}{4 \rho^{2}+4|x|^{2} \rho+\gamma^{4}|t|^{2}}+2 \gamma^{2}|t|^{2}=V_{1} R_{2}^{-1} V_{2}^{\mathrm{T}} .
\end{aligned}
$$

再注意到由 (4.1) 有

$$
\frac{H}{\rho}=\frac{1}{|x|^{2} \rho+2 \gamma^{2}|t|^{2}},
$$

从而, 当 $x \neq o$ 时, $\operatorname{det}\left(\mathbb{X}^{l} \mathbb{X}^{r} \phi(x, t)\right) \neq 0$ 当且仅当

$$
\begin{aligned}
0 \neq Q\left(\beta, \gamma^{2}\right)=1 & -\frac{\beta+2}{|x|^{2} \rho+2 \gamma^{2}|t|^{2}}\left[\frac{4|x|^{2} \rho^{3}}{4 \rho^{2}+4|x|^{2} \rho+\gamma^{4}|t|^{2}}+\gamma^{2}|t|^{2}+\frac{1}{|x|^{4}} \frac{4 \rho^{2}+\gamma^{4}|t|^{2}+4|x|^{2} \rho}{4 \rho^{2}+\gamma^{4}|t|^{2}}\right. \\
& \left.\times\left(\frac{4|x|^{4} \rho^{2}}{4 \rho^{2}+4|x|^{2} \rho+\gamma^{4}|t|^{2}}+2 \gamma^{2}|t|^{2}\right)^{2}\right] \\
= & 1-\frac{\beta+2}{\rho_{1}+2 \varsigma}\left[\frac{4 \rho_{1}^{3}}{4 \rho_{1}^{2}+4 \rho_{1}+\gamma^{2} \varsigma}+\varsigma+\frac{4 \rho_{1}^{2}+\gamma^{2} \varsigma+4 \rho_{1}}{4 \rho_{1}^{2}+\gamma^{2} \varsigma}\left(\frac{4 \rho_{1}^{2}}{4 \rho_{1}^{2}+4 \rho_{1}+\gamma^{2} \varsigma}+2 \varsigma\right)^{2}\right],
\end{aligned}
$$

506 
此处,

$$
\varsigma=\gamma^{2} \frac{|t|^{2}}{|x|^{4}}, \quad \rho_{1}=\frac{1}{2}(1+\sqrt{1+4 \varsigma}) .
$$

注意到由 (4.1) 有 $\rho_{1}^{2}=\rho_{1}+\varsigma$. 令 $\theta=\frac{\varsigma}{\rho_{1}} \geqslant 0$ 得

$$
\begin{aligned}
Q\left(\beta, \gamma^{2} ; \theta\right) & =1-\frac{\beta+2}{1+2 \theta}\left[\frac{1+\theta}{2+\left(1+\frac{\gamma^{2}}{4}\right) \theta}+\theta+(1+\theta) \frac{2+\left(1+\frac{\gamma^{2}}{4}\right) \theta}{1+\left(1+\frac{\gamma^{2}}{4}\right) \theta}\left(\frac{1}{2+\left(1+\frac{\gamma^{2}}{4}\right) \theta}+2 \theta\right)^{2}\right] \\
& =1-\frac{\beta+2}{1+2 \theta}\left[\frac{1+\theta}{2+\kappa \theta}+\theta+(1+\theta) \frac{2+\kappa \theta}{1+\kappa \theta}\left(\frac{1}{2+\kappa \theta}+2 \theta\right)^{2}\right],
\end{aligned}
$$

其中 $\kappa=1+\frac{\gamma^{2}}{4}>1$.

显然, $Q$ 在区域 $\left(\beta, \gamma^{2}, \theta\right) \in \mathbb{R}^{+} \times \mathbb{R}^{+} \times[0,+\infty)$ 上连续, $Q\left(\beta, \gamma^{2} ; 0\right)<0$; 当 $(\beta, \theta)$ 固定时, $Q$ 为 $\gamma^{2}>0$ 的严格单调递增函数. 我们也注意到当 $\gamma^{2} \leqslant 4$ 时, 有

$$
Q\left(\beta, \gamma^{2} ; \theta\right) \leqslant-\frac{\beta}{2}<0, \quad \forall \theta \geqslant 0, \quad \lim _{\gamma^{2} \rightarrow+\infty} \sup _{\theta \geqslant 0} Q\left(\beta, \gamma^{2} ; \theta\right)=1 .
$$

记

$$
4 \leqslant D(\beta)=\sup \left\{\gamma^{2} ; Q\left(\beta, \gamma^{2} ; \theta\right)<0, \forall \theta \geqslant 0\right\}<+\infty .
$$

通过一些繁琐、初等的计算, 我们发现当 $0<\gamma^{2} \leqslant 16+20 \sqrt{3}$ 时, 有 $Q\left(0, \gamma^{2} ; \theta\right) \leqslant 0, \forall \theta \geqslant 0$. 因此, 我 们有 $D(\beta) \geqslant 16+20 \sqrt{3}$.

\section{参考文献}

1 Fefferman C, Stein E M. $H^{p}$ spaces of several variables. Acta Math, 1972, 129: 137-193

2 Fefferman C. Inequalities for strongly singular convolution operators. Acta Math, 1970, 124: 9-36

3 Hirschman I I. Multiplier Transforms I. Duke Math J, 1959, 26: 222-242

4 Wainger S. Special Trigonometric Series in k-Dimensions. Mem Amer Math Soc, 59. Providence, RI: Amer Math Soc, 1965

5 Giulini S, Meda S. Oscillating multipliers on noncompact symmetric spaces. J Reine Angew Math, 1990, 409: 93-105

6 Alexopoulos G. Oscillating multipliers on Lie groups and Riemannian manifolds. Tôhoku Math J, 1994, 46: 457-468

7 Li H Q. Estimations du noyau de la chaleur sur les variétés coniques et ses applications. Bull Sci Math, 2000, 124: 365-384

8 Laghi N, Lyall N. Strongly singular integral operators associated to different quasi-norms on the Heisenberg group. Math Res Lett, 2007, 14: 825-838

9 Lyall N. Strongly singular convolution operators on the Heisenberg group. Trans Amer Math Soc, 2007, 359: 4467-4488

10 Bonfiglioli A, Uguzzoni F. Nonlinear Liouville theorems for some critical problems on H-type groups. J Funct Anal, 2004, 207: 161-215

11 Kaplan A. Fundamental solutions for a class of hypoelliptic PDE generated by composition of quadratic forms. Trans Amer Math Soc, 1980, 258: 147-153

12 Cowling M, Dooley A H, Korányi A, et al. H-type groups and Iwasawa decompositions. Adv Math, 1991, 87: 1-41

13 Del Hierro M. Dispersive and Strichartz estimates on H-type groups. Studia Math, 2005, 169: 1-20

14 Hebisch W, Zienkiewicz J. Multiplier theorem on generalized Heisenberg groups, II. Colloq Math, 1995, 69: 29-36

$15 \mathrm{Hu}$ J Q, Li H Q. Gradient estimates for the heat semigroup on H-type groups. Potential Anal, 2010, 33: $355-386$

16 Korányi A. Geometric properties of Heisenberg type groups. Adv Math, 1985, 56: 28-38

17 Li H Q. Estimations optimales du noyau de la chaleur sur les groupes de type Heisenberg. J Reine Angew Math, 2010, 646: 195-233

18 Li H Q, Qian B. Centered Hardy-Littlewood maximal functions on Heisenberg type groups. Trans Amer Math Soc, 2014, 366: 1497-1524 
19 Liu H P, Wang Y Z. A restriction theorem for the H-type groups. Proc Amer Math Soc, 2011, 139: 2713-2720

20 Lust-Piquard F. Riesz transforms on generalized Heisenberg groups and Riesz transforms associated to the CCR heat flow. Publ Mat, 2004, 48: 309-333

21 Müller D, Ricci F, Stein E M. Marcinkiewicz multipliers and multi-parameter structure on Heisenberg (-type) groups, II. Math Z, 1996, 221: 267-291

22 Randall J. The heat kernel for generalized Heisenberg groups. J Geom Anal, 1996, 6: 287-316

23 Tan K H, Yang X P. Characterisation of the sub-Riemannian isometry groups of H-type groups. Bull Austral Math Soc, 2004, 70: 87-100

24 Beals R, Gaveau B, Greiner P C. Hamilton-Jacobi theory and the heat kernel on Heisenberg groups. J Math Pures Appl (9), 2000, 79: 633-689

25 Li H Q. Fonctions maximales centrées de Hardy-Littlewood sur les groupes de Heisenberg. Studia Math, 2009, 191: $89-100$

26 Stein E M, Murphy T S. Harmonic Analysis: Real-variable Methods, Orthogonality, and Oscillatory Integrals. Princeton Mathematical Series, 43. Monographs in Harmonic Analysis, III. Princeton, NJ: Princeton University Press, 1993

\section{Strongly singular convolution operators on the Heisenberg-type groups}

\section{HongQuan}

Abstract In this article, we obtain $L^{2}$ estimates for some strongly singular convolution operators on the Heisenberg-type groups. In particular, our results improve and generalize those obtained in the setting of Heisenberg groups by Laghi and Lyall. Furthermore, some simpler and more effective techniques are introduced in this article.

Keywords strongly singular convolution operators, Heisenberg-type group, Korányi norm

$\operatorname{MSC}(2010) \quad 42 \mathrm{~B} 20,43 \mathrm{~A} 80$

doi: 10.1360/N012013-00105 